\title{
Molecular Analysis of Hybrids among the Ornamental Eucalypts Eucalyptus macrocarpa, E. pyriformis, and E. youngiana
}

\author{
Kirsty Neaylon, Kate L. Delaporte, Margaret Sedgley, ${ }^{1}$ and Graham G. Collins \\ Department of Horticulture, Viticulture and Oenology, Adelaide University, Waite Campus, P.M.B. 1, \\ Glen Osmond, S.A. 5064, Australia
}

John G. Conran

Department of Environmental Biology, Adelaide University, S.A. 5005, Australia

\begin{abstract}
AdDitional INDEX words. Eucalyptus, hybrid paternity, RAPD-PCR, UPGMA, multivariate analysis, DNA fingerprinting, genetic similarity

Aвstract. The potential for hybridization among three species of Eucalyptus L'Hér in the Series Macrocarpae, E. macrocarpa Hook (Mottlecah), E. pyriformis Turcz. (pear-fruited mallee), and E. youngiana F. Muell. (large-fruited mallee), was investigated using molecular data generated by randomly amplified polymorphic DNA (RAPD)-polymerase chain reaction (PCR) analysis. Samples of DNA from seedlings derived from controlled pollinations, and from different individuals from each species, were amplified with six different 10-mer primers. The presence or absence of RAPD fragments was used to generate a dendrogram based on genetic similarity, an ordination derived by multidimensional scaling (MDS), and a minimum spanning tree (MST) to show the relative links and dissimilarities between the individuals tested. Two clusters were identified on the unweighted pair-group method arithmetric average dendrogram. The first included all of the $E$. macrocarpa genotypes and all but one of the $E$. macrocarpa hybrids. The second included all of the $E$. youngiana and $E$. pyriformis genotypes and their hybrids. The MDS ordinations placed the hybrid seedlings between the parent species. From the 30 progeny investigated, 28 were assessed from the molecular data to be hybrids from controlled pollinations. The remaining two seedlings appeared to be derived from self-pollination. The parentage of two mature trees, thought to be natural hybrids involving the three species, was also investigated. One was confirmed as a cross between $E$. youngiana and $E$. pyriformis, but the second was less certain because of its low genetic similarity to all other individuals, and may be a hybrid involving species not included in this study.
\end{abstract}

To be successful commercially, ornamental trees should possess features such as attractive flowers, unique and colorful fruits, unusually shaped or colored foliage, or an attractive trunk and bark. One or more of these features is present in many eucalypts, particularly those in the Series Macrocarpae, which includes Eucalyptus macrocarpa (Mottlecah), E. pyriformis (pear-fruited mallee), and E. youngiana (large-fruited mallee). These three species comprise some of the most striking ornamentals, with large showy flowers up to $4 \mathrm{~cm}$ in diameter, and colored stamens.

Identification of eucalypt species and determination of possible hybrid status is achieved by measurements of plant form and bark type, and the morphology and color of foliage, flowers, fruit, and seeds (Williams and Brooker, 1997). However, the characters of closely related species are often similar and may be further altered by environmental factors, tree maturity, and seasonal variability in flowering times. Amplification of specific sequences in genomic DNA has recently been used to produce unique fingerprints of individuals of a range of genera that are independent of these factors, and provides an ideal method of identification (Mekuria et al., 1999). Thus, identification of eucalypts and the occurrence and parental determination of hybrids could be achieved using molecular methods.

Received for publication 26 June 2000. Accepted for publication 27 Dec. 2000 We thank Jennifer Gardner for access to the Waite Arboretum and State Flora and ForestrySA for access to the Monarto Woodland. Support for this study was provided by the Noel and Vivian Lothian Scholarship in Ornamaental Horticulture. The cost of publishing this paper was defrayed in part by the payment of page charges. Under postal regulations, this paper therefore must be hereby marked advertisement solely to indicate this fact.

${ }^{1}$ To whom reprint requests should be addressed: e-mail: margaret.sedgley@ adelaide.edu.au.
The primary aim of this study was to determine the degree of hybridization that could occur among the three ornamental eucalypt species, E. macrocarpa, E. youngiana, and E. pyriformis, using molecular techniques. A secondary aim was to determine whether two ornamental eucalypts located in the Waite Arboretum, Urrbrae, South Australia, were hybrids. This study contributes to an ongoing program to develop eucalypts as ornamentals (Ellis et al., 1991, Wirthensohn et al., 1999).

\section{Materials and Methods}

Plant materials. Forty-seven trees were included in the analysis. Five trees of each of the three species E. macrocarpa, $E$. youngiana, and E. pyriformis were selected from the Waite Arboretum and the Laidlaw Plantation, of the Waite Campus of the University of Adelaide, Urrbrae, South Australia, and the Monarto Woodland, Callington, South Australia. Preference was given to those plants used as parents in the controlled pollinations (Delaporte, 2000). Two putative natural hybrids between $E$. youngiana and E. pyriformis and E. youngiana and E. macrocarpa (designated hybrid 1 and 2, respectively) were selected in the Waite Arboretum, Urrbrae, South Australia. In addition, five seedlings from each possible combination in both directions from controlled pollinations between the three species were selected. These seedlings demonstrated morphological characters that were intermediate between those of the parents as determined by multivariate analysis (Delaporte, 2000).

RAPD TECHNIQUE. Fully expanded leaves were collected and stored at $-20{ }^{\circ} \mathrm{C}$ until required. DNA was extracted using the protocol reported by Lamboy and Alpha (1998). The quality of the DNA was estimated by calculating the ratios of absorbances 
at 260 and $230 \mathrm{~nm}$ and at 260 and $280 \mathrm{~nm}$ (Mekuria et al., 1999). DNA samples with values of 1.7 or higher were used for further analysis.

Fifteen decamer oligodeoxyribonucleotide primers (Operon Technologies, Alameda, Calif.) were screened using DNA extracted from one representative from each of the three parent species. Six of these primers (A01, A07, B05, B12, D05 and D06) were selected on the basis of those revealing the highest number of clear, polymorphic bands. These same six primers were used on all parents and hybrid combinations, both natural and artificial.

Polymerase chain reactions (PCRs) were performed using protocols described by Bradley et al. (1996) and Wirthensohn et al. (1999). The PCR program consisted of an initial denaturation of $2 \mathrm{~min}$ at $94^{\circ} \mathrm{C}$, followed by 41 cycles of $1 \mathrm{~min}$ at $94^{\circ} \mathrm{C}, 1 \mathrm{~min}$ at $36^{\circ} \mathrm{C}, 2 \mathrm{~min}$ at $72{ }^{\circ} \mathrm{C}$, with a final extension step of $5 \mathrm{~min}$ at 72 ${ }^{\circ} \mathrm{C}$. Amplified DNA fragments were mixed with $10 \times$ loading buffer and separated on $1.5 \%$ Seakem genetic technology grade (GTG) agarose gels in Tris-borate electrophoresis (TBE) buffer for $40 \mathrm{~min}$ at a constant current of $80 \mathrm{~mA}$. A 100 base pair (bp) molecular-weight marker ladder (GeneWorks, Adelaide, Australia) was loaded into wells on each side of the gel to aid interpretation of band identity. PCR products were stained with ethidium bromide $\left(0.5 \mu \mathrm{g} \cdot \mathrm{mL}^{-1}\right)$, visualized under ultraviolet (UV) light, and photographed with Polaroid 667 film (Polaroid, Hertfordshire, U.K.). Digital images were captured with Tekcap (Version 1.0, Tekram Corp., Fremont, Calif.) and visualized with Paint Shop Pro (Version 5.0, Jasc Software Inc., Minneapolis, Minn.). Duplicate and if necessary triplicate amplifications were conducted to ensure reproducible results. All reproducible bands were used for data analysis.

Data AnAlysis. Digital images of the gels were analyzed using Gel-Pro Analyzer (Version 3.1, Media Cybernetics, Silver Spring, Md.) based on the sizes of fragments in the $100 \mathrm{bp}$ DNA ladder. PCR products were scored as present (1) or absent (0) for all individuals, ignoring faint or nonreproducible bands, and the data recorded in a binary matrix.

Binary matrices were analyzed using the program NTSYS-pc (Numerical Taxonomy and Multivariate Analysis System, Version 2.02j, Exeter Software, Setauket, N.Y.). Genetic similarities between the different plants were obtained by performing pairwise comparisons of all individuals using the simple matching coefficient (Bradley et al., 1996). Cluster analysis was performed using the unweighted pair-group method arithmetric average (UPGMA), a widely used clustering algorithm providing equal

Fig. 1. Dendrogram showing genetic similarity between 47 eucalypt individuals of Eucalyptus macrocarpa, E. pyriformis, and E. youngiana as artificial and putative hybrids, using six primers, the simple matching coefficient, and UPGMA clustering $(\mathrm{Em}=$ E. macrocarpa, $\mathrm{Ep}=$ E. pyriformis, $\mathrm{Ey}=$ E. youngiana, and PutHy = putative hybrid). weighting between individuals as they fuse to form groups (Belbin, 1991, 1994), and a dendrogram indicating genetic similarities was constructed.

Nonhierarchical distance multivariate analysis with multidimensional scaling (ordination) was generated from the binary matrices with the PATN computer analysis program (Belbin, 1994). Genetic dissimilarities between individuals or operational taxonomic units (OTUs) were calculated using Gowers Metric (MC), generating an association matrix between the objects (ASO). Gowers Metric, a range standardized Manhattan distance, corresponds to the simple matching coefficient when used for presence or absence of data such as the binomial data generated from molecular analysis (Belbin, 1994). The resulting matrix was analyzed using UPGMA.

Semistrong hybrid multidimensional scaling ( $\mathrm{SSH}$ ) was used to ordinate the ASO dissimilarities with 100 repeats to reduce the effects of localized suboptimal minima (Nicolle and Conran, 1999). The stress level was recorded, indicating the level of distortion applied by the SSH to the dendrogram. The resultant ordination points were plotted as a three dimensional scatter plot (Sigmaplot 4.0 for Windows, SPSS Science, Chicago, Ill.).

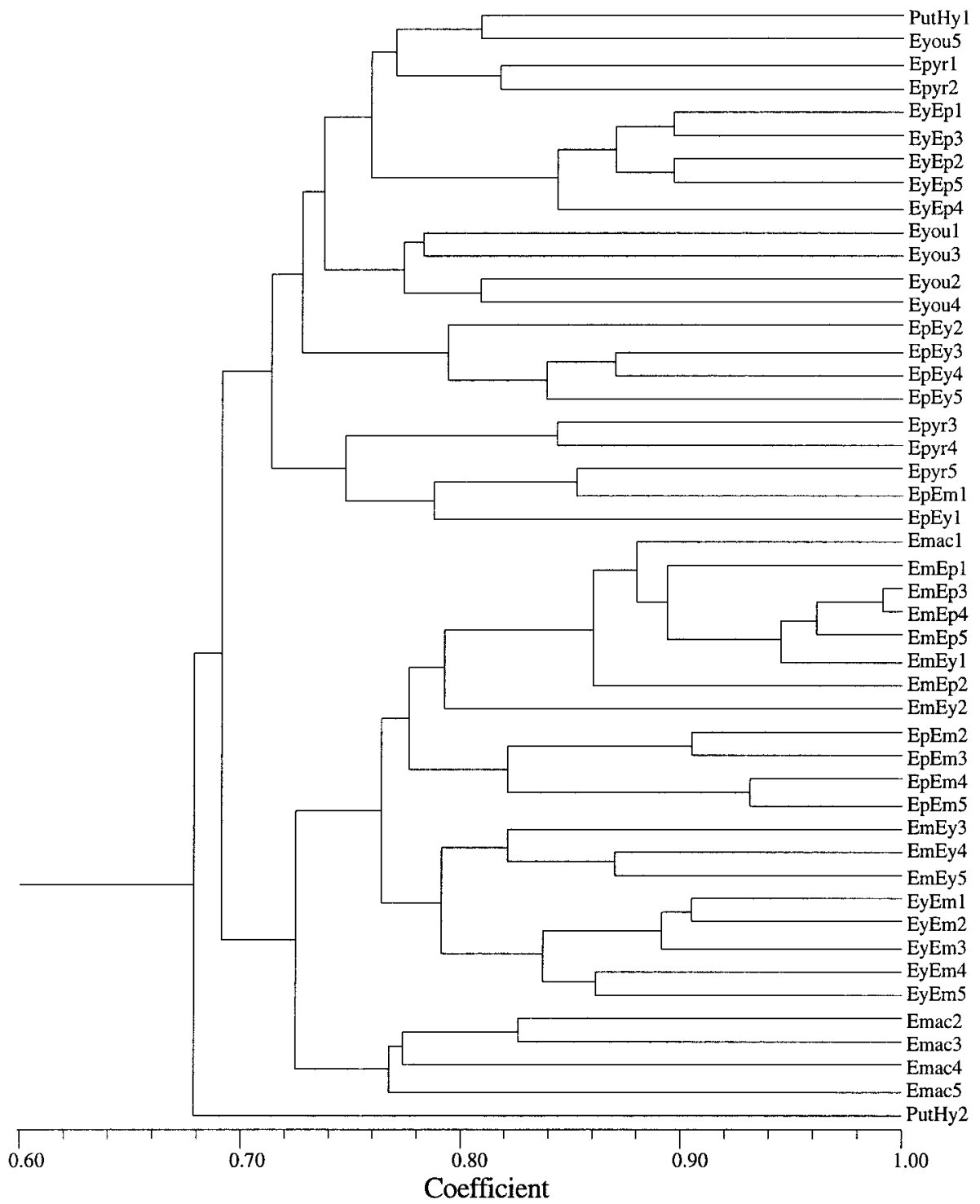




\section{Results}

RAPD POLYMORPHISMS. The six decamer primers selected for RAPD-PCR analysis produced a total of 115 bands, of which 92 were polymorphic and the remaining 23 monomorphic. Individual primers produced between 16 and 22 bands varying in size from 270 to $3000 \mathrm{bp}$.

HieraRChiCal Distance analysis. The dendrogram from the analysis of 47 eucalypt individuals after RAPD-PCR is shown in Fig. 1. There were two clusters with $69 \%$ similarity. One cluster included the five genotypes of E. macrocarpa as well as all but one seedling plant from controlled pollinations involving $E$. macrocarpa. The second cluster contained the five genotypes of both E. pyriformis and E. youngiana, as well as all seedlings from controlled pollinations between the two species, E. pyriformis and E. youngiana. Putative hybrid 1 was also associated with this cluster. Putative hybrid 2 was distinct from both clusters with a genetic similarity of $68 \%$.

Within the E. macrocarpa cluster, there were five subclusters, four of which were very consistent in their complement. One included all but one of the E. macrocarpa genotypes. Another included all of the $E$. youngiana $\times$ E. macrocarpa hybrids. Three of the five E. macrocarpa $\times$ E. youngiana hybrids comprised one subcluster, with four of the five E. pyriformis $\times$ E. macrocarpa hybrids in another subcluster. The more variable subcluster included all of the E. macrocarpa $\times$ E. pyriformis hybrids, two of the E. macrocarpa $\times$ E. youngiana hybrids, and one of the $E$. macrocarpa genotypes.

The E. pyriformis/E. youngiana cluster also included five subclusters, three of which were consistent in their complement. One included all of the $E$. youngiana $\times E$. pyriformis hybrids, another included all but one of the $E$. youngiana genotypes, and another all but one of the $E$. pyriformis $\times E$. youngiana hybrids. One of the two variable subclusters included three of the five E. pyriformis genotypes, and one each of $E$. pyriformis $\times$ E. youngiana and $E$. pyriformis $\times$ E. macrocarpa hybrids. The other included the remaining two E. pyriformis genotypes, the remaining one E. youngiana genotype and putative hybrid 1.

NonHIERARCHICAL DISTANCE aNALYSIS. The SSH ordination of all individuals into three dimensions (Fig. 2) showed 11 point clusters-E. macrocarpa $\times$ E. youngiana, E. pyriformis X E. macrocarpa, E. macrocarpa, $E$. macrocarpa $\times$ E. pyriformis, putative hybrid 2, E. pyriformis, E. pyriformis $\times$ E. youngiana, putative hybrid $1, E$. youngiana, E. youngiana $\times$ E. pyriformis, and E. youngiana $x$ E. macrocarpa, similar to the clusters identified by the dendrogram. The stress level for the ordination is $19.6 \%$, which is within the maximum acceptable level of $20 \%$ (Belbin, 1994).

The three species, E. macrocarpa,E. pyriformis, and $E$. youngiana formed individual groups clearly distinct from each other. All of the seedlings that were generated when E. macrocarpa was used as either the male or female parent (except for one E. pyriformis $\times$ E. macrocarpa outlier) formed distinct groups, which had close associations with the E. macrocarpa group. The E. youngiana X E. macrocarpa seedling group, although most closely associated with the E. macrocarpa group, showed intermediacy with the E. youngiana group. Similarly, the group containing the E. pyriformis $\times$ E. youngiana seed- lings showed intermediacy between both species; whereas the $E$. youngiana $\times$ E. pyriformis seedlings formed a group which was more closely associated with the E. youngiana cluster.

The high degree of overlap shown in the ordination plot reflects the close relationship between the three species and shows that seedlings located intermediate between adult groups with some degree of overlap are most likely to be hybrids between the two species.

\section{Discussion}

This study has shown that hierarchical and nonhierarchical analysis of molecular data confirmed the success of both natural and artificial interspecific hybridization between the three $E u c a$ lyptus species, E. macrocarpa, E. pyriformis, and E. youngiana. From the thirty progeny investigated in this study, 28 were assessed to be hybrids from controlled pollinations, while the remaining two appear to be derived from self-pollination. Although flowers were bagged immediately after emasculation, the detection of self-pollinated progeny indicates that some pollen was probably shed during removal of the anthers.

Eucalyptus macrocarpa and its putative hybrids formed a genetic cluster distinct from crosses involving E. youngiana and E. pyriformis, indicating that E. macrocarpa can be more readily distinguished genetically from the other two species. All seedlings derived from E. macrocarpa in the controlled pollinations have a closer genetic similarity with E. macrocarpa than with any other parent. The only exception to this is the Ep x Em1 seedling

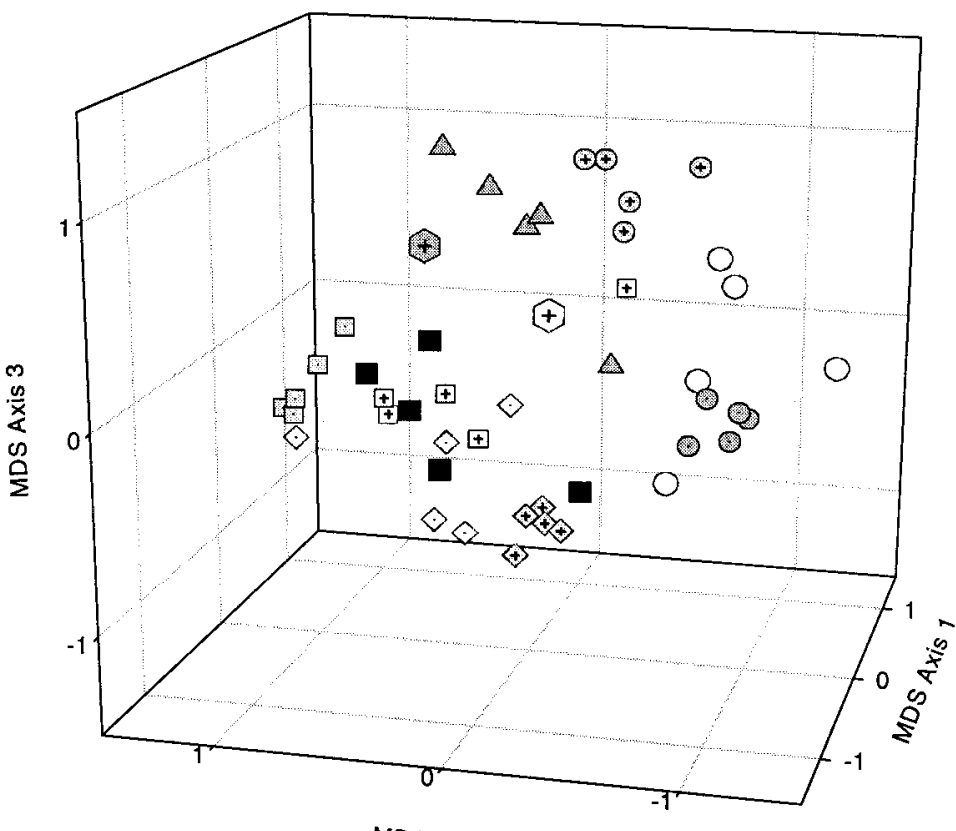

MDS Axis 2

\begin{tabular}{|ll|}
\hline & E. macrocarpa \\
0 & E. pyriformis \\
$\oplus$ & E. youngiana \\
Putative hybrid 1 \\
Putative hybrid 2 \\
$\odot$ EmxEp \\
$\odot$ EmxEy \\
0 EpxEy \\
$\odot$ EyxEp \\
$\oplus$ EyxEm \\
$\oplus$ EpxEm
\end{tabular}

Fig. 2. Ordination analysis (hybrid multidimensional scaling) of 47 eucalypt individuals. The stress level is $19.6 \%(\mathrm{Em}=$ Eucalyptus macrocarpa, $\mathrm{Ep}=$ E. pyriformis, and Ey = E. youngiana). 
which grouped within the E. youngiana and E. pyriformis cluster. Ep $\times$ Em1, and also Ep $\times$ Ey1, are linked directly to E. pyriformis 5 , which was the female parent used in the cross, indicating that these seedlings may be derived from self-pollination rather than cross-pollination. Although eucalypts preferentially outcross, thereby favoring pollination from another source, they are also commonly self-compatible (Sedgley, 1989).

Hybrid eucalypts generally show characteristics that are intermediate between the parent species, unless there is an expression of allelic dominance (Ashton and Sandiford, 1988). The clustering of E. macrocarpa hybrid seedlings closer to E. macrocarpa, regardless of whether $E$. macrocarpa was used as the male or female parent, indicates that E. macrocarpa displays genetic dominance over both E. youngiana and E. pyriformis. Although seedlings from controlled pollinations involving $E$. macrocarpa cluster closest to the E. macrocarpa individuals, nonhierarchical analysis of all individuals showed that these seedlings form a group intermediate between the parental species. Such clustering of offspring closest to one or both parents is an indicator of hybrid status (McDade, 1997). All E. macrocarpa seedlings have genetic similarities closer to $E$. macrocarpa 1 , than to the other four E. macrocarpa individuals. This is because E. macrocarpa 1 was used as a pollen source for the artificial hybridizations, in addition to being the female parent in all controlled pollinations involving this species. Hybrids can also be distinguished from within the $E$. macrocarpa cluster as belonging to a particular cross. For example, seedlings of crosses between $E$. youngiana and $E$. macrocarpa form a cluster with a genetic similarity of $\approx 83 \%$, and distinct from all other individuals.

The hierarchical analysis grouped E. pyriformis and E. youngiana to a cluster separate from E. macrocarpa. Within this cluster, hybrids where E. youngiana was used as the female parent formed a group close to the $E$. youngiana representatives. Similarly, hybrids where E. pyriformis was used as the female parent, grouped closest to the E. pyriformis representatives. Seedlings from crosses involving $E$. youngiana and $E$. pyriformis appear to be dominated by genes from the female rather than the male parent, indicating that neither species displays dominance over the other. Nonhierarchical analysis of individuals from molecular data indicates that seedlings from controlled pollinations between $E$. pyriformis and $E$. youngiana display characters intermediate between their parental species. The three $E$. pyriformis individuals (E. pyriformis $3, E$. pyriformis 4 , and $E$. pyriformis 5) located at the Monarto Woodland formed a cluster with genetic similarity of only $71 \%$ to the other E. pyriformis individuals, indicating a high degree of genetic diversity between certain individuals. Similarly, E. youngiana trees located at Monarto Woodland and the Waite Arboretum are genetically closer than $E$. youngiana 5 located in the Laidlaw plantation. Seed for $E$. youngiana 5 was obtained from a commercial supplier and is most likely to have been collected from native stands, whereas the source of the other E. youngiana trees is unknown. Eucalyptus youngiana is sparsely distributed from Western Australia to
South Australia, which may account for the diversity identified within the species.

A second objective of this study was to determine the most likely identity of two highly ornamental putative hybrids located in the Waite Arboretum. Putative hybrid 1 is most probably a hybrid between $E$. pyriformis and E. youngiana. For putative hybrid 2 , its low genetic similarity of $68 \%$ to all other individuals suggests that this tree is not representative of any of the three species investigated. Other related species with the potential to form interspecific hybrids, such as E. drummondii Benth. or $E$. sessilis (Maiden) Blakely (Griffin et al., 1988), may be the parental species of putative hybrid 2 .

\section{Literature Cited}

Ashton, D.H. and E.M. Sandiford. 1988. Natural hybridisation between Eucalyptus regnans F. Muell. and E. macrorhyncha F. Muell. in the Cathedral Range, Victoria. Austral. J. Bot. 36:1-22.

Belbin, L. 1991. The analysis of pattern in bio-survey data, p. 176-190. In C.R. Margules and M.P. Austin (eds.). Nature conservation: Cost effective biological surveys and data analysis. Commonwealth Scientific and Industrial Research Organisation, Canberra, Australia.

Belbin, L. 1994. PATN pattern analysis package V3.5. Commonwealth Scientific and Industrial Research Organisation, Division of Wildlife and Ecology, Canberra, Australia.

Bradley, K.F., M.A. Rieger, and G.G. Collins. 1996. Classification of Australian garlic cultivars by DNA fingerprinting. Austral. J. Expt. Agr. 36:613-618.

Delaporte, K.L. 2000. Eucalypts for ornamental horticulture: selection, interspecific hybridisation and postharvest testing. $\mathrm{PhD}$ thesis, Dept. of Horticulture, Viticulture and Oenology, Adelaide University, South Australia.

Ellis, M.F., M. Sedgley, and J.A. Gardner. 1991. Interspecific pollenpistil interaction in Eucalyptus L'Hér. (Myrtaceae): The effect of taxonomic distance. Ann. Bot. 68:185-194.

Griffin, A.R., I.P. Burgess, and L. Wolf. 1988. Patterns of natural and manipulated hybridisation in the genus Eucalyptus L'Hérit.-A review. Austral. J. Bot. 36:41-66.

Lamboy, W.F. and C.G. Alpha. 1998. Using simple sequence repeats (SSRs) for DNA fingerprinting germplasm accessions of grape (Vitis L.) species. J. Amer. Soc. Hort. Sci. 123:182-188.

McDade, L.A. 1997. Hybrids and phylogenetic systematics III. Comparison with distance methods. Systematic Bot. 22:669-683.

Mekuria, G.T., G.G. Collins, and M. Sedgley. 1999. Genetic variability between different accessions of some common commercial olive cultivars. J. Hort. Sci. Biotechnol. 74:309-314.

Nicolle, D. and J.G. Conran. 1999. Variation in the Eucalyptusflocktoniae complex (Myrtaceae) and the description of four new taxa from Southern Australia. Austral. Systematic Bot. 12:207-239.

Sedgley, M. 1989. Ovule and seed development in Eucalyptus woodwardii Maiden (Symphyomyrtus). Bot. Gaz. 150:271-280.

Williams, J.E. and M.I.H. Brooker. 1997. Eucalypts: An introduction, p. 1-15. In J.E. Williams and J.C.Z. Woinarski (eds.). Eucalypt ecology: Individuals to ecosystems. Cambridge Univ. Press, Cambridge, U.K. Wirthensohn, M.G., G.G. Collins, G.P. Jones, and M. Sedgley. 1999. Variability in waxiness of Eucalyptus gunnii foliage for floriculture. Scientia Hort. 1385:1-11. 